Article

\title{
Improving Power and Resource Management in Heterogeneous Downlink OFDMA Networks
}

\author{
Nalliyanna Goundar Veerappan Kousik ${ }^{1}\left(\mathbb{D}\right.$, Yuvaraj Natarajan ${ }^{2}$, Kallam Suresh ${ }^{3}(\mathbb{D}$, \\ Rizwan Patan ${ }^{4, *}$ and Amir H. Gandomi ${ }^{5}$ \\ 1 School of Computing Science and Engineering, Galgotias University, NCR, Delhi 201307, India; \\ nvkousik@galgotiasuniversity.edu.in \\ 2 Research and Development, ICT Academy, Chennai 600096, India; yuvaraj@ictacademy.in \\ 3 Department of Computer Science \& Engineering, Sree Vidyanikethan Engineering College, Tirupati 517102, \\ India; sureshkallam@vidyanikethan.edu \\ 4 Department of Computer Science \& Engineering, Velagapudi Ramakrishna Siddhartha Engineering College, \\ Vijayawada 520007, India \\ 5 Faculty of Engineering \& Information Systems, University of Technology Sydney, Ultimo, NSW 2007, \\ Australia; gandomi@uts.edu.au \\ * Correspondence: rizwan@vrsiddhartha.ac.in
}

Received: 12 February 2020; Accepted: 31 March 2020; Published: 10 April 2020

\begin{abstract}
In the past decade, low power consumption schemes have undergone degraded communication performance, where they fail to maintain the trade-off between the resource and power consumption. In this paper, management of resource and power consumption on small cell orthogonal frequency-division multiple access (OFDMA) networks is enacted using the sleep mode selection method. The sleep mode selection method uses both power and resource management, where the former is responsible for a heterogeneous network, and the latter is managed using a deactivation algorithm. Further, to improve the communication performance during sleep mode selection, a semi-Markov sleep mode selection decision-making process is developed. Spectrum reuse maximization is achieved using a small cell deactivation strategy that potentially identifies and eliminates the sleep mode cells. The performance of this hybrid technique is evaluated and compared against benchmark techniques. The results demonstrate that the proposed hybrid performance model shows effective power and resource management with reduced computational cost compared with benchmark techniques.
\end{abstract}

Keywords: power management; sleep mode selection; resource management; deactivation algorithm

\section{Introduction}

Significant voice and data requirements have placed pressure on wireless radio links and the infrastructure of wireless networks globally. Hence, serving an increasing number of users has become a major challenge for network operators even if the bandwidth is wider. Several solutions for wireless communications have been previously proposed; however, they are ineffective in terms of capital and operational costs. Other techniques, such as cell densification on heterogeneous wireless networks, reduced the cost of small cell deployment with enhanced coverage and capacity [1]. However, the distribution of unplanned small cells is associated with serious threats that lead to coand cross-tier interference [2]. Densification of small cells leads to co-tier interference and generates multiple overlapped cell boundaries [3]. Macrocells cause extreme cross-tier interference and leads to performance degradation of low power cells [4]. Such interference issues are avoided through radio resource management (RRM), which coordinated intercells through the allocation of users orthogonally in the frequency and time domains, where interference is avoided [5]. 
Fractional frequency reuse (FFR) is another intercell interference coordination (ICIC) technique that resolves the problem of lesser complexity and minimal cooperation between the base stations. Thus, using FFR achieves the throughput rate and coverage between the cell-edge users and spectral efficiency [6]. Further, in a multi-tier heterogeneous network that includes macro, outdoor pico-, and indoor femto- base stations, FFR avoids density problems due to co-tier interference [7]. However, resource allocation for different channel conditions is an impossible task in the FFR technique. Similar to ICIC, range expansion (RE) is used to improve the channel capacity via off-loading macrocell users to low power picocells. Unfortunately, RE degrades the throughput [8]. To improve optimal performance gains, Radio Resource Management (RRM) [9] and joint RRM [10] techniques were improved with new policies [11].

One such policy is sleep mode in a heterogeneous network that improves capacity and power efficiency. Overall, $10 \%-60 \%$ of power is saved using the sleep mode technique [12]. Small cells in heterogeneous networks can operate either in sleep mode (SL) or ready mode (RE). During SL, certain parts of small cell hardware are switched off, while other parts operate at low power. The sleep mode decision in hardware is determined by algorithms. The user/small cell/core network controls the transition from SL to RE and vice versa. In SL mode, the small cells turn off the transmission when there is no active user with low-power capability. In the core network with SL mode, backhaul controls the transition between the states in the core. In user-controlled SL mode, the user activates the cells through broadcast signals [12]. However, traffic arrival [13] from various nodes increases the discrepancy of inefficient power consumption in small cells. To overcome such issues, the semi-Markov decision process is used as a decision-maker in sleep mode selection (SLS) for improved performance [14].

Sleep mode selection (SLS) optimizes several aspects of wireless cellular networks. The semi-Markov decision (SMD) process for improved SLS in a wireless network uses a policy optimization algorithm for solving the stochastic optimization problem. With this policy optimization, the Quality of Service (QoS) maintains better energy consumption levels [15]. For improved switching, a semi-Markov decision with a lightweight algorithm solves the problem of policy optimization and achieves a better runtime SLS. In addition, the optimal power saving class is used to maximize power efficiency in small cell heterogeneous networks [14]. In [16], SLS was implemented in time and spatial domains at different traffic loads. Fewer antenna without SLS performed well, but the entire antenna in a heterogeneous network with SLS outperformed the system without SLS. In [17], a many-to-one decentralized algorithm was developed that consists of a matching user association algorithm with resource block allocation, where a sleep-voting mechanism was used in macrocells. In [18], binary exponential SLS was used for power consumption in an Institute of Electrical and Electronics Engineers (IEEE) 802.16e/m system's mobile station. This method considered the residual battery energy for improving the consumption of energy in mobile stations. Improved power consumption in heterogeneous networks leads to reduced Signal Input to Noise Ratio (SINR), which uses offloading [19], RRM [20], cooperative RRM [21], and mixed-integer RRM [22]. However, in [23], SLS was activated on a single-tier per available frequency or time resource, which reduced the network power consumption with increased SINR and coverage in macrocells. In [24], this was further extended to a three-way SLS for the stable and efficient operation of a digital subscriber line (DSL). This increased the energy-delay Pareto optimal tradeoff using stable SLS operation as compared to conventional strategies with SLS. Furthermore, a sleep mode best effort distribution heuristic algorithm was proposed in [25] for reducing power consumption in base stations.

The techniques used in proposed SL strategies exploit the idle time to minimize power consumption in heterogeneous networks. Here, for the improved consumption of power, small cells with an inactive user are set to SL mode through SLS. To further improve power consumption in the small cell heterogeneous orthogonal frequency-division multiple access (OFDMA) network, a hybrid SLS-based RRM is adopted. This engages FFR to leverage the gain (both rate and capacity). The ad hoc behavior in femtocell positioning is exploited with a minimal user to examine the probability of a cell's temporary sleep mode. This acts as an additional degree of freedom that optimizes the obtainable resources. Interference is reduced to achieve this; however, poor placement of small cells allows increased 
utilization of resources. The ICI is controlled through the incorporation of resource partitioning (RP) with small cell/users' orthogonalization. The reuse is reduced with orthogonalization using a selection algorithm that identifies the base station (BS) with the highest reduction in reuse. The information is fed into an RP scheme to determine whether the deactivation of a small cell can improve resource utilization. The hybrid method improves the throughput and power consumption with reduced computation cost. Also, the hybrid facilitates improved deactivation decision behavior with limited transmission between the core and BSs. A suitable mathematical model analyses the performance of the hybrid model to reveal superior performance and a closer match between the simulated and analytical results.

The main contributions of the hybrid model include:

- Design of a hybrid model that identifies interferences during which the switching off or sleeping mode of some small cells can enhance the capacity of the heterogeneous network.

- Formulation of an SLS mode-based RRS for providing an enhanced gain of reused resources.

- Presentation of a model that captures the interference received and the resultant performance of upper bounds accurately verifies the estimated gains.

The remainder of the paper is structured as follows. Section 2 provides a brief description of the network model and its assumptions. Sections 3 and 4 thoroughly discuss the hybrid model. Results and discussions are presented in Section 5. Final remarks are presented in Section 6.

\section{Interference Map (IM) Approach}

An IM identifies the conflicts [25] in a heterogeneous system. The measurement report (MR) from UE is used to build the IM, and the MR is triggered by a radio resource control (RRC) message. For the determination of physical cell identity (PCI), UE is used to scan the nearby BS and the power reference signal that is received (reported to serving BS). The local IM, $\zeta_{x}$, of serving BS $x$ is expressed as:

$$
\zeta_{x}(z, y)= \begin{cases}0 & \frac{F_{x, z}}{F_{y, z}}>\beta \\ 1 & \frac{F_{x, z}}{F_{y, z}}<\beta\end{cases}
$$

where

$z$ is referred to as the user served by $x$ and its nearby BS $y$.

$\beta$ is referred to as the signal to interference (SIR) threshold.

$F_{x, z}$ is referred to as the power received from BS $x$ to UE $z$,

$F_{j, k}$ is referred to as the interference power received from BS $y$ to UE $z$.

The threshold value is selected based on the optimization objective. Typically, a higher threshold maximizes the performance, and a lower threshold maximizes the throughput. If the local IM is updated by BS, then local IM $\zeta_{x}$ is sent to Femtocell Management System (FMS), iff $\zeta_{x}^{\prime}$, which is different from local IM $\zeta_{x}[25]$.

$$
\sum_{z=1}^{S_{x}} \sum_{x=1}^{B}\left|\zeta_{x}(z, x)-\zeta_{x}^{\prime}(z, x)\right|>0
$$

The universal IM of the heterogonous network with $\zeta \in \mathfrak{R}^{Z \times B}$ is represented as:

$$
\begin{array}{cc}
\zeta(z, x)=w, & x \text { serves BS } k \\
\zeta(z, x)=0 & y \text { doesn } / \text { interfere } z \\
\zeta(z, x)=1 & y \text { interferes } z
\end{array}
$$

where $w$ is a positive integer number $\notin 1$ and 0. 


\section{The Proposed Method}

The main objective is to identify whether deactivating some definite small cells can improve the wider perspective of the network. Here, the centralized controller is selected as FMS due to unplanned deployment of femtocells. Determining the deactivation of such potential cells can result in improvements to the capacity and wide reuse of frequency. Attention is given to the re-association of network users to relocate to the nearby cells during the deactivation process. This resolves the co-tier interferences through orthogonal RRB. The scenario of a cell being switched off is shown in Figure 1.

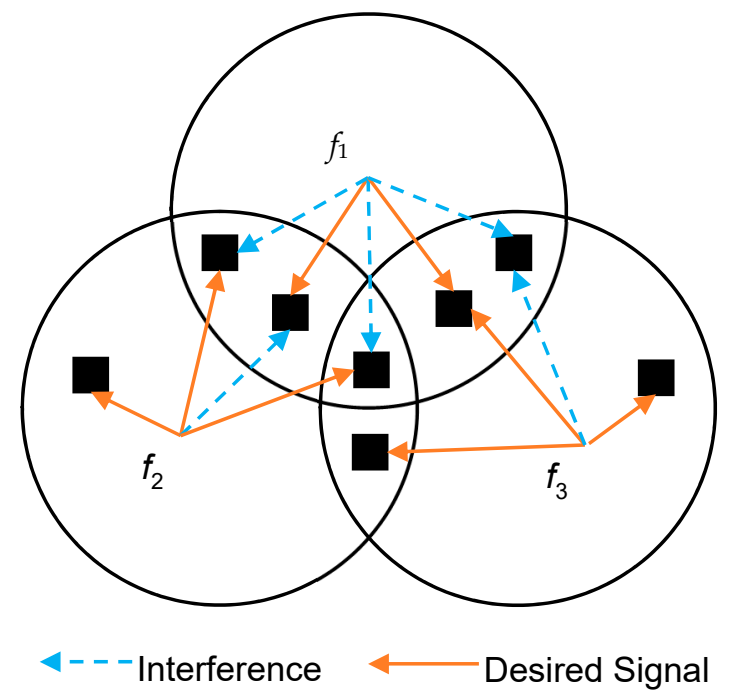

Figure 1. A scenario of a cell switching off.

\subsection{Sleep Mode for Maximizing Capacity}

The stochastic optimization problem is formulated using the semi-Markov SLS (SMD-SLS) decision process that considers MU density, spatial distribution, and scheduling in a heterogeneous network. The SMD-SLS model achieves a favorable trade-off between performance and power consumption in multi-cell heterogeneous networks. The stochastic optimization problem is solved through an optimal set of power scheduling policies. Finally, an iterative algorithm solves the SMD-SLS-based stochastic optimization and yields an optimal decision for minimizing power consumption in BS.

The main objective involves analyzing the maximum capacity in a network after the deactivation of the BS set. This leads to the formulation of the capacity maximization problem using the semi-Markov SLS (SMD-SLS) decision process. Consider a BS to be in SL or RE and that the BSs state is indicated by a variable $\varphi_{x} \in\{0,1\}$; where, $\varphi_{x}=0$ denotes the SL state and vice versa. The attainable capacity, $c_{k, n}$, using a semi-Markov SLS (SMD-SLS) decision at a physical resource block is expressed as:

$$
c_{z, n}=W \cdot \log \left(Y_{z, n}+1\right)
$$

where $W$ denotes the physical resource block bandwidth $n$, which is similar to all physical resource blocks. The capacity maximization problem is thus formulated with constraint functions and a stochastic reward that minimizes the consumption of power with respective performance constraints. The SMD-SLS model is applied using a state-space definition and activation functions.

The SMD-SLS state, $N_{x}(t)$, at time $t$ is characterized by the density of each cell. The location of the user state is $L_{x}(t)$ and each cell's transmit power state is $E_{x}(t)$. Then, the SMD-SLS state of cell $x$ is $s_{x}(t)=\left\{N_{x}(t), L_{x}(t), E_{x}(t)\right\}$ and the probability matrix of state transition is $\left[\delta_{s x s^{\prime} x}(t)\right]=\left[\psi_{x}(t), \Theta_{x}(t)\right.$, $\left.\Phi_{x}(t)\right] \cong \varphi(t) \phi(t) \psi(t)$. The SMD-SLS state transition probability changes from the state $s_{x}$ to state $s_{x}^{\prime}$.

The state-space of the SMD-SLS model is denoted as $S \cong\{D, L, E\}$. The activation state is $A$, which is denoted by $A=\left\{a_{1}(t), a_{2}(t), a_{3}(t), a_{4}(t)\right\}$, where $a_{1}(t)$ is the action of turning off the FMS without changing 
the nearby femtocells, $a_{2}(t)$ is the action of turning off the FMS and increasing the transmission power of two nearby femtocells by a multilevel power control in FSS, $a_{3}(t)$ is the action of unchanged FMS and increasing the transmission power of two nearby femtocells by a multilevel power control in FSS, and, finally, $a_{4}(t)$ is the action of decreasing the power of FSS due to reduced MU without changing nearby cells. The current state of SMD-SLS, $s(t) \in S$, and action $a(t) \in A$ represent the probability matrix of state transition for the next state $s(t+1)$, which is $\mathrm{P}_{\mathrm{r}}\{a(t), s(t+1) \mid s(t)\}$. This Markovian function probability represents the state transition of the previous state and is independent of the next stage when specified in the present stage.

In SMD-SLS, during each epoch, power control scheduling decides the power allocation to the femtocells, as per the power and traffic profiles. Power control scheduling uses the activation function $a(t)$ at each epoch. Since the objective is to minimize power consumption, a reward function is used as the power consumption function for the femtocells. Thus, the reward function is defined as:

$$
R(S, A)=1 / P(S, A)
$$

where $P(S, A)$ is the total power consumed by all BSs that acts as a function of the SMD-SLS state and SMD-SLS activation for obtaining optimal trade-off between performance and energy efficiency.

Thus, an optimal policy is selected, denoted by $\pi$, which has maximized the total reward under the threshold constraint with SINR function on the $z$ th user's SINR $g_{k}(x)$ within $x$. This is formulated as in Equation (6):

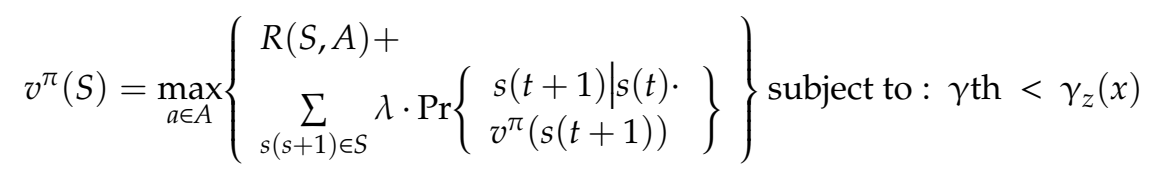

where:

$v^{\pi}(S)$ is referred to as the maximum rewards under the policy $\pi$ over the present state $S$, $g$ th is referred to as the targeted SINR, and $\lambda$ is referred to as the discount factor $g_{k}(x)$ is referred to as the SINR of $z$ user under cell $x$.

$$
\gamma_{z}(x)=\frac{\eta \cdot P_{t x}(x)}{\left[d_{z}(x)\right]^{\xi}\left(N_{0}+\sum_{y=1, y \neq x}^{N} P_{t x}(y)\right)}
$$

where:

$h$ is referred to as the propagation coefficient, $P_{t x}(x)$ is referred to as the transmit power of cell $x$, $x$ is referred to as the attenuation coefficient, which is set between 2 and 5 , $d_{z}(x)$ is referred to as the distance from user $z$ to BS cell $i$, $N_{0}$ is referred to as the AWGN function.

$$
\begin{gathered}
\max _{\phi, x} \sum_{x=1}^{B} \sum_{z=1}^{Z} \sum_{n=1}^{N} \phi_{i} c_{z, n} \chi_{z, n} \text { s.t. } \sum_{z=1}^{Z} \chi_{z, n} \leq 1, \forall x, n \\
\sum_{n=1}^{N} \chi_{z, n}=\Psi_{k} \forall z
\end{gathered}
$$


where:

$\chi_{k, n}=1$ when user $z$ is allocated in $n$ resource block, and vice versa.

$\Psi_{k}$ represents the total resources allocated to user $z$.

Equation (8) assures that each resource block is allocated to a user within the same femtocell. Equation (9) guarantees that $z \mathrm{UE}$ is allocated with the $z$ resource block. Equation (7) is an integer linear programming problem (ILP) that is solved by ILP solvers.

Efficient methods are required to solve the running time of ILP solvers, which is uncertain, thereby leading to computational complexity. To reduce this, a definite solution is provided that possess two parts. In the initial part, the FMS with a definite set of criteria determines the BSs for deactivation. Additionally, resource partitioning with the condition of the selected BS's switching state determines whether resource utilization can be increased. The final part is the allocation of resources that is independently solved by/within each BS using the Channel Quality indicator (CQI) user reports. Thus, the output of the initial part consists of an active BS set and an allocated resource block within a permissible level per user. Therefore, the orthogonal property is ensured with resource partitioning, which determines the best resources allocated per user.

\subsection{Iteration Algorithm}

An iterative algorithm is used to solve the problem of optimization that is specified by Equation (7). Specifically, the iterative algorithm derives the optimal policy and the expected reward function. The algorithm is expressed as:

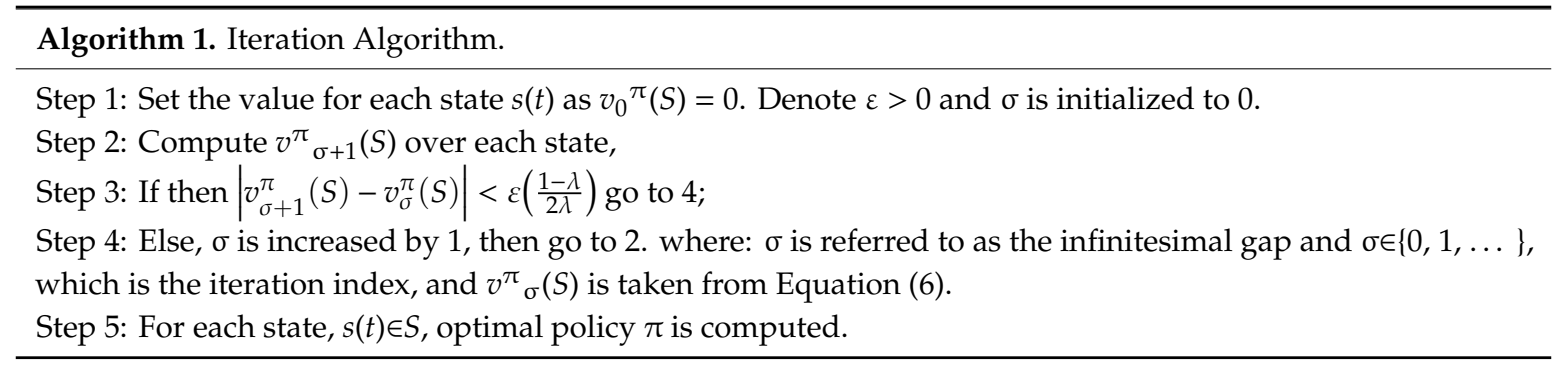

The computational complexity is thus calculated as $O\left(\|S\|^{2}\|A\|\right) ;\|\cdot\|$ represents cardinality set.

\subsection{Femtocell Deactivation Condition}

The BS selection is performed using the femtocell deactivation condition. The deactivation trials are described as follows:

Trial-1: The total number of conflicts (NoC) of an individual BS can be inferred as total UEs interfered with by BS $x$. The total number of $x$ connected UEs is interfered with through neighboring BSs. Hence, the NoC is calculated by the FMS, which is associated with individual BS based on IM:

$$
N o C_{x}=\sum_{y=1 \neq x}^{B} \sum_{z=1}^{S_{x}}\{\zeta(z, y)=1\}+\sum_{z=1}^{Z}\{\zeta(z, x)=1\}
$$

where $x=a$ and $x=b$; if $a=b$ then $x=1$ and $a \neq b$ then $x=0$.

Trial-2: If entire UEs belong to BS, then the UE proportion located in the handover region is expressed as:

$$
H_{x}=\frac{\sum_{z=1}^{S_{i}} L_{x}}{S_{x}}
$$


where

$H_{x}$-handover ratio of BS $x$,

$S_{x}$-total UEs linked to $x$

$L_{x}$-total UEs served by $x$, which is located inside the region overlapped with nearby femtocells.

Trial-3: The total UEs connected to $x$, which is expressed as:

$$
S_{x}=\sum_{z=1}^{Z}\{\zeta(z, x)=w\}
$$

Trial-4: The total received power (its sum) of all UEs connected to $x$ is expressed as:

$$
P_{x}=\sum_{z=1}^{S_{x}}\left[F_{x, z}\right]
$$

Trial-5: The interference received power (its sum) by $x$ from nearby BSs is expressed as:

$$
I_{x}=\sum_{z=1}^{S_{x}} \sum_{y=1 \neq x}^{B}\left[F_{y, z}\right]
$$

\subsection{Deactivation Algorithm}

The deactivation algorithm for small cells incorporates a deactivation scheme and a resource partitioning scheme. Deactivating the selected or unwanted BSs reduces the interference with increased resource reuse and capacity. Measurements are taken to prevent outage probability of BSs, which are connected to cells in sleep mode. The deactivation algorithm for small cells is performed as follows:

- Prior to executing the tests for deactivation, the sum of resource blocks allocated to each user is determined and compared with the updated deactivated value after deactivation policy.

$$
\Psi_{\text {tot }}=\sum_{x=1}^{B} \sum_{z=1}^{S_{x}} \sum_{n=1}^{N} A_{z, n} \cdot \chi_{z, n}
$$

where $\Psi_{\text {tot }}$ and $\Psi^{\prime}{ }_{\text {tot }}$ - present and previous resources utilized, respectively.

- The reuse efficiency is significantly affected by NoC, which is applied with Trial-1 to determine the maximum NoC in BSs:

$$
\wp=\operatorname{maximum}(\mathrm{NoC})
$$

where $\wp$-preferred BS.

- If $\wp=1$, BS is switched off, or else if $\wp>2$, then BS having maximum handover ratio is applied with Trial-2:

$$
\wp=\operatorname{maximum}(H)
$$

- If $\wp=0$, which means BS is not nominated in the handover region, the deactivation policy is selected based on Trial-4 for all BSs with equal users:

$$
\wp=\operatorname{minimum}(P)
$$

Equation (18) is used to find whether $\wp$ possesses less received power than other BSs and whether it enhances the overall capacity. Otherwise, Trial-3 selects the BS with minimum users for reducing the UEs dropping:

$$
\wp=\operatorname{minimum}(S)
$$


- The dropping UEs are avoided by selecting the largest handover ratio on $\wp$, which is located between the other cells within the handover region. If $\wp>1$ and $\wp=\mathrm{H}$, and if users lie within the handover region of nearby cells, Trial-5 is used for selecting $\wp$, which is shown below:

$$
\wp=\operatorname{minimum}(I)
$$

Trial-5 in Equation (20) selects a BS that receives the maximum interference from neighboring BSs, because such UEs achieve enhanced signal quality when handed over to the cells. Otherwise, the selection of $\wp$ is based on Equation (18).

- Finally, $\wp$ is deactivated and the nearby BSs in an active state with maximum power receive disconnected users. However, a user will be dropped if it lies outside the coverage range.

- $\quad \zeta$ is updated and then the $\Psi_{\text {tot }}$ is calculated.

- The $\Psi_{\text {tot }}$ (updated state) is compared with the $\Psi^{\prime}{ }_{\text {tot }}$ (previous state) and $\wp=$ SL mode if $\Psi_{\text {tot }}>$ $\Psi^{\prime}{ }_{\text {tot }}$. The process continues to searching for the other users/BSs for deactivation.

\subsection{Allocation of Resource}

Each BS/user is adopted independently using the frequency resource allocation algorithm that is measured from total resource per user. BSs use the CQI report from each user for selecting the preferred resource block and-after determination of the preferred resource block-an update is periodically sent to BSs. The resource management allocates user data packets in the frequency domain according to the implemented scheduling rule using reinforcement learning for improving the QoS provisioning for the frequency resource allocation algorithm towards improving the QoS of BSs.

\section{Performance Analysis}

In order to study the impact of a downlink signal-to-interference ratio (SIR) on the user location with certain required parameters, the simulation results can be verified using the probability density function (PDF). To order to model accurately the received interference, a grid design of static BS structures and random users is presumed.

\subsection{Signal to Interference Model}

The received interference power from BS, $x$, to UE, $z$, is $P_{t} d_{x, z}^{-\varphi}$, where $P_{t}$ represents the transmission power of $x, \phi$ represents the path loss exponent and $d_{x, z}$ represents the distance between $x$ and $z$. Assume that the value $P_{t}$ is similar for all BSs and, hence, the user $z$ is not interfered with by $y$ when the interference of $y<$ threshold:

$$
P_{t} d_{x, z}^{-\varphi}<\frac{P_{t} d_{x, z}^{-\varphi}}{\beta} \text { or } \frac{d_{x, z}}{d_{y, z}}<\left(\frac{1}{\beta}\right)^{\frac{1}{\varphi}}
$$

where $\beta$ represents the threshold of SIR, and $d_{x, z} / d_{y, z}$ or $D$ is the ratio of the distance between $z, x$, and $y$. Finally, the user $k$ is detected if $(1 / \beta)^{1 / \varphi} \leq D \leq 1$ and it is not detected when it lies in $0<D<(1 / \beta)^{1 / \varphi}$. Conflict Analysis

The deactivation decision is majorly impacted through NoC since the grid scenario is considered with BSs $(F)$ and UEs $\left(S_{f}\right)$, which is connected with BS $(f)$. The total interfered users in the detected coverage BS $(f)$ area are given as:

$$
L_{a}=S_{f}(F-1)\left[\operatorname{Pr}\left(\left(\frac{1}{\beta}\right)^{\frac{1}{\varphi}} \leq D_{x, y} \leq 1\right)\right]
$$


or

$$
L_{a}=S_{f}(F-1)\left\{1-F_{D_{x, y}}\left(\frac{1}{\beta}\right)^{\frac{1}{\varphi}}\right\}
$$

where $F_{D}$ is the cumulative density function (CDF) of $f_{D}$.

Further, the total users of ' $f$ ' interfered with through its nearby small cells is:

$$
L_{b}=S_{f}\left\{1-F_{D_{x, y}}\left(\frac{1}{\beta}\right)^{\frac{1}{\varphi}}\right\}
$$

and

$$
N o C_{f}=L_{a}+L_{b}
$$

Figure 2 shows their effect of the SIR threshold on NoC between the nominated cell and neighboring cells under different distance criteria. As shown in Figure 2, NoC is proportional to the SIR threshold, as NoC increases with the increase in the SIR threshold. A greater number of interfered users is detected within a coverage area by increasing the NoC.

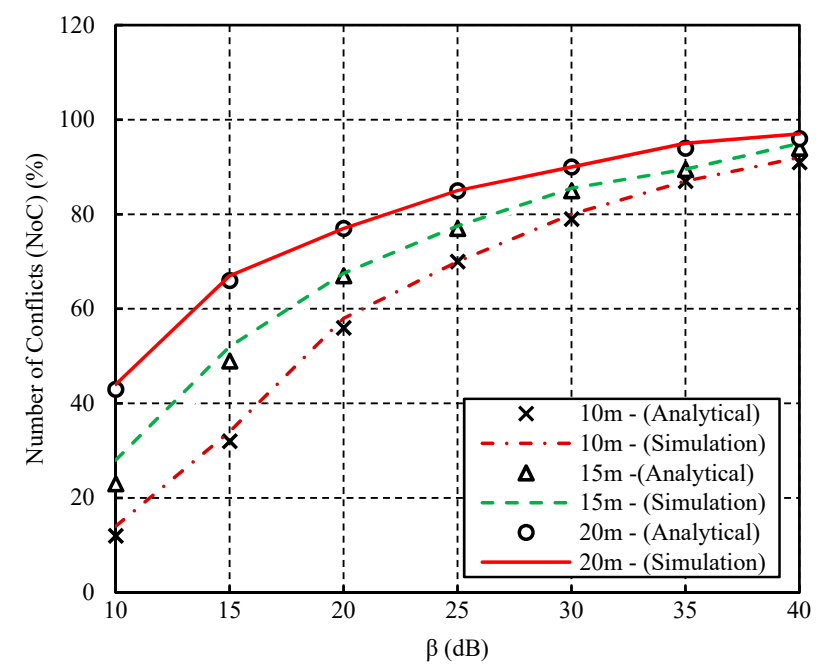

Figure 2. Signal to interference (SIR) threshold impact on number of conflicts (NoC).

\subsection{Reuse Efficiency}

The SIR threshold $(\beta)$ effect completely influences reuse efficiency and, hence, the $\beta$ effect on utilization of resources using PDF is studied in this section. Consider a system with $f$ detected users. $S_{f}$ users per $F$ BSs is equal to:

$$
\tau_{f}=S_{f}+S_{f}(F-1)\left\{1-F_{D_{x, y}}\left(\frac{1}{\beta}\right)^{\frac{1}{\varphi}}\right\}
$$

Total resources available per user is $\Psi_{z}=N / T_{f}$ and total resources allocated per BS is $\Omega_{f}=k / S_{f}$, as follows:

$$
\Omega_{f}=\frac{N}{1+\left((F-1)\left\{1-F_{D_{x, y}}\left(\frac{1}{\beta}\right)^{\frac{1}{\varphi}}\right\}\right)}
$$

The impact of SIR threshold on reuse efficiency is shown in Figure 3; it can be inferred that reuse efficiency decreases as the SIR threshold increases, since NoC is found to be higher in the network. Hence, it can be seen that the SIR poses a serious threat to reuse efficiency due to NoC constraints. 


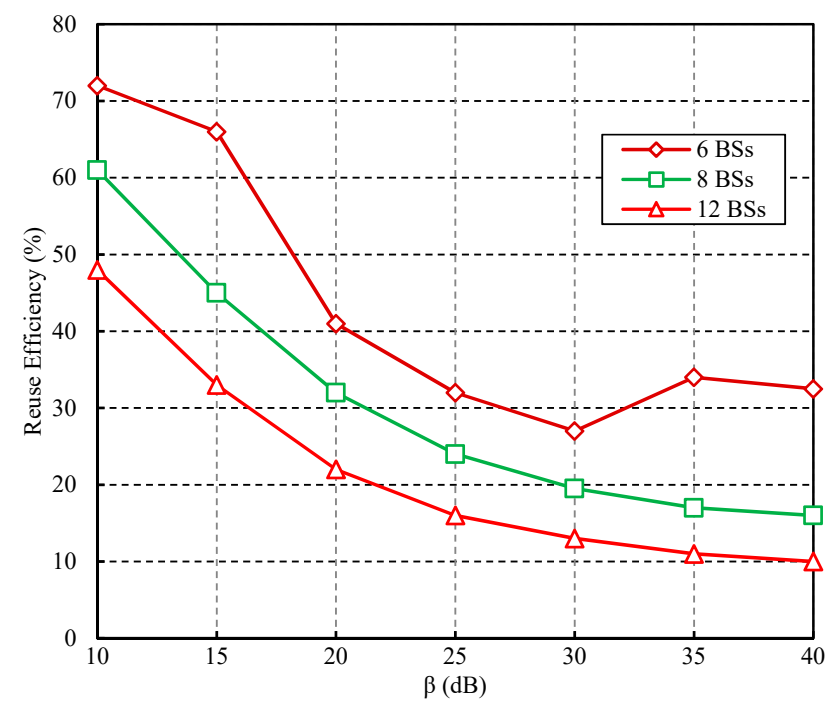

Figure 3. SIR impact on reuse efficiency.

Signaling Overhead

The total exchanged information between the BSs and the centralized FMS is:

$$
d_{s}=d_{f_{i}}+d_{c}
$$

where $d_{f_{i}}$ represents the total bits for sending the IM from BS $f_{i}$ to centralized FMS.

Assume $f_{i}$ BS with neighboring $J_{i}$ BSs and $S_{i}$ users, then:

$$
d_{f_{i}}=J_{i} \cdot d_{m} \cdot S_{x}
$$

where $d_{m}$ represents the total bits to encode the IM.

The centralized FMS requires the feedback of the local IM from its individual BS, which extracts required information including $\mathrm{NoC}, H$, and $S$. If $\wp>1$, then the signal power received by BSs of the users is sent as feedback to the centralized FMS:

$$
d_{c}=\tau \cdot d_{t} \cdot S_{x}
$$

where $d_{t}$ represents deactivating the $\wp$ and $\tau$ represents the total bits required to send the feedback of CQI (4 bits). Conversely, the FMS is restricted in sending the feedback to the BSs, since allocation is independently performed by each individual BS.

\subsection{Computational Complexity}

An optimal and exhaustive benchmark approach uses an iterative mechanism to check the impact of BS deactivation with the available resources. In contrast, the proposed hybrid method selects the optimal BSs without the need of an exhaustive mechanism. The exhaustive approach requires $O(M)$ iterations to find the deactivated cell whereas the proposed hybrid approach does not use such a mechanism to find the deactivated cell. $M$ defines the overall BSs for testing over $M$ iterations with $M$ possibilities.

\section{Performance Evaluation}

This section presents the metrics of homogeneous and heterogeneous solution. The parameters for simulation are presented in Table 1. 
Table 1. Parameters.

\begin{tabular}{cc}
\hline Parameter & Value \\
\hline Resource block & $30 / 50$ \\
Bandwidth & $7 \mathrm{MHz}$ \\
Carrier $f_{c}$ & $2.4 \mathrm{GHz}$ \\
Period of OFDMA symbol & $1.5 \times 10^{-4} \mathrm{~s}$ \\
Channel & Rayleigh \\
Wall loss $(w l)$ & $18 \mathrm{~dB}$ \\
Pathloss $(\phi)$ & 3 \\
Shadowing $\left(X_{\epsilon}\right)$ & $8 \mathrm{~dB}$ \\
Tx power in macro-cell & $46 \mathrm{dBm}$ \\
Tx power in femtocell & $20 \mathrm{dBm}$ \\
Macro-femto minimum separation & $75 \mathrm{~m}$ \\
Macro-UE minimum separation & $35 \mathrm{~m}$ \\
Macrocell radius & $289 \mathrm{~m}$ \\
\hline
\end{tabular}

The proposed system is analyzed by a comparative evaluation with the Manchester technique [25]. Both methods use IM for indicating the interference between the users and the BSs. The Manchester technique makes a similar assumption as SMD-SLS except for the use of the SMD algorithm. The proposed method is also compared to the optimal approach with upper bound performance.

Figure 4 shows the mean rate at various cell densities, which reveals that the proposed method is significantly better than the Manchester method. The results in Figure 4 show that there is a significant improvement in reuse efficiency due to reduced interference in neighboring cells. This is due to the minimization of the number of femtocells that are unnecessarily active. The performance gap between the proposed SMD-SLS and Manchester techniques is increased with a denser network formation, where improvements have increased to $23 \%, 22 \%$, and $25 \%$ for a certain number of femtocells, for example, 12, 13 and 14, respectively. Furthermore, the performance of the Feedback Shift Register (FSR)-Manchester method shown in Figure 4 approaches the performance of the Manchester technique with increasing cell density since, in the FSR-Manchester technique, the entire resources are allocated to BSs, and the users are protected from interference, thereby tending to yield a higher data rate than the Manchester method with ICI.

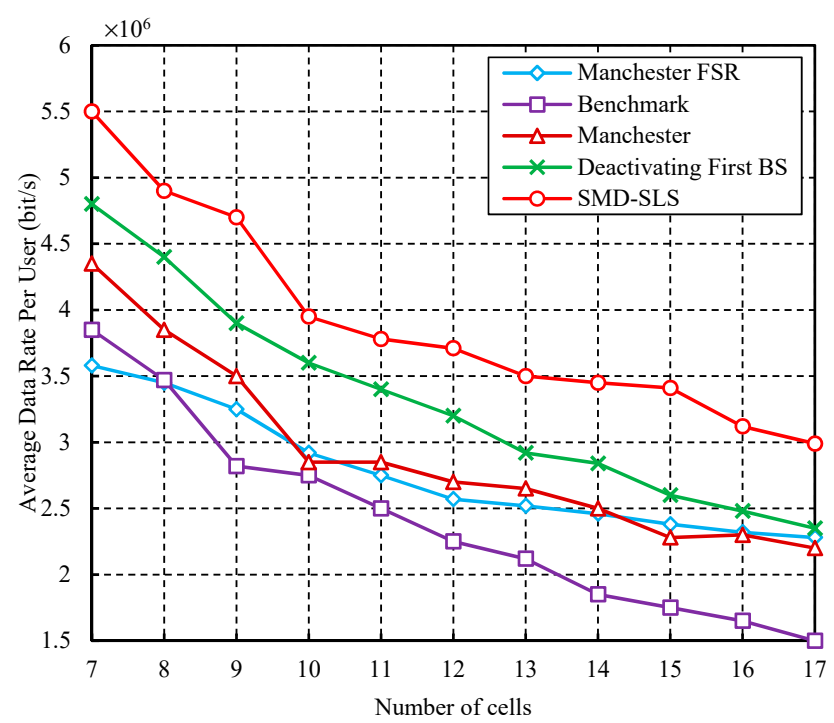

Figure 4. Comparison of average data rate per user with different cell densities.

Figure 5 shows the Energy Consumption Rating (ECR) with various cell densities, which indicates that there is an improvement in the data rate as compared to the existing method. The performance of 
ECR in the proposed SMD-SLS is better than in the Manchester method, even with higher densities, since the aim of the benchmark technique [26] is to reduce the consumption of energy.

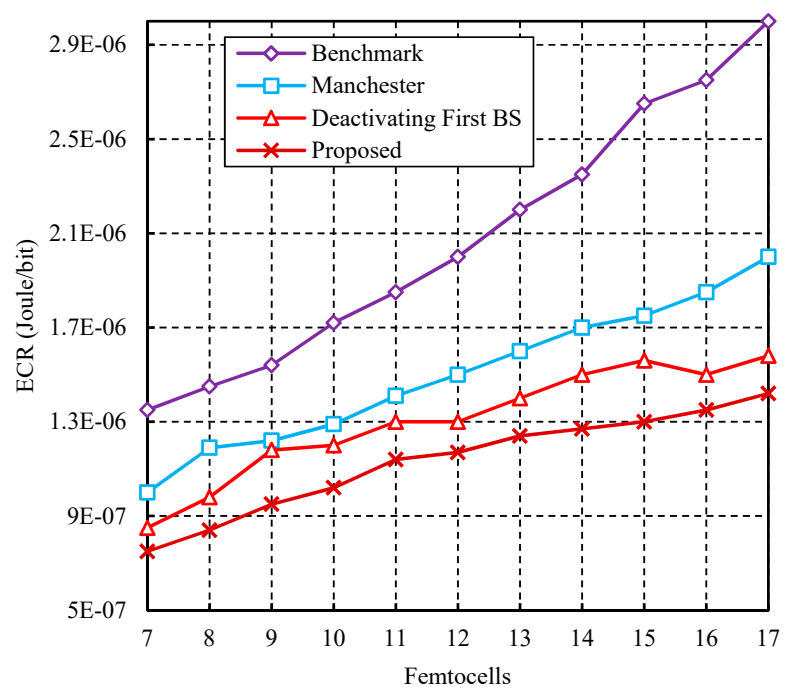

Figure 5. Comparisons of ECR (Jolues/bit) with different cell densities.

Figure 6 shows a comparison of power consumption between the SMD-SLS technique and the other methods. As can be seen in Figure 6, the proposed SMD-SLS technique consumes less power than the Manchester technique. This is because switching off the hardware parts during SL mode further reduced power consumption in the proposed technique [12]. Alternatively, it is found that the benchmark method consumes low power as compared to the other methods, since additional BSs are further switched off.

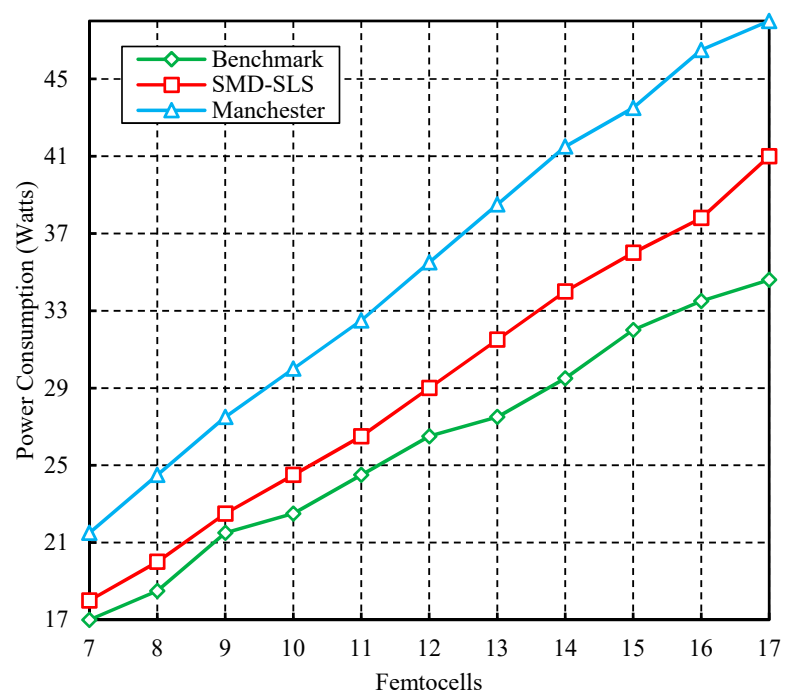

Figure 6. Comparisons of power consumption for different cell densities.

An evaluation of throughput vs CDF with a total of seven deployed femtocells is shown in Figure 7. The throughput results of the proposed SMD-SLS technique are better than the results of the Manchester technique, with a rate increase of approximately $30 \%-32 \%$. The overall interference rate is significantly reduced due to activation of the SL mode in unnecessary femtocells. The avoidance of such disturbances further improved resource utilization by the nearby BSs. The BS receives a stronger signal due to the open-access policy of BSs with the users. Total deactivated cells in all three techniques 
are shown in Figure 8, in which the highest rate of deactivation occurred in the proposed SMD-SLS technique compared to the benchmark and Manchester technique. The dropping ratio of total users vs total UEs is shown in Figure 9, which shows that the proposed SMD-SLS technique displayed an increased dropped ratio with increasing user density, since dropping UEs are reduced against the Manchester technique. The average capacity of UEs (shown in Figure 10) using the proposed method is improved by a rate of $14 \%-20 \%$ compared to the Manchester technique, based on total BSs.

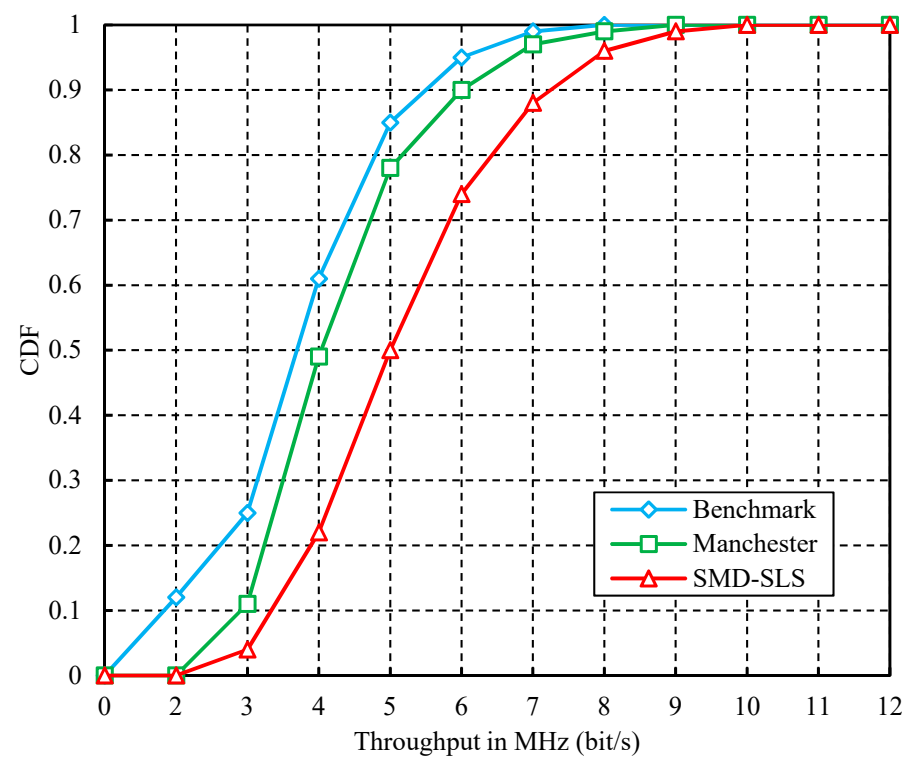

Figure 7. Average throughput vs cumulative density function (CDF) deployed with seven cells.

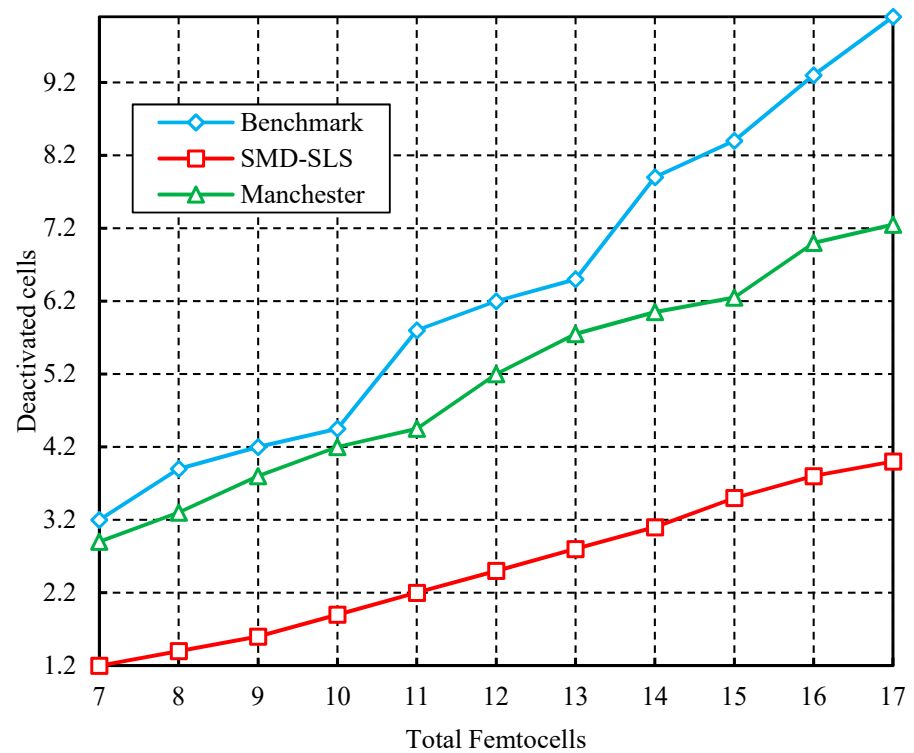

Figure 8. Deactivated cells in a number of femtocells. 


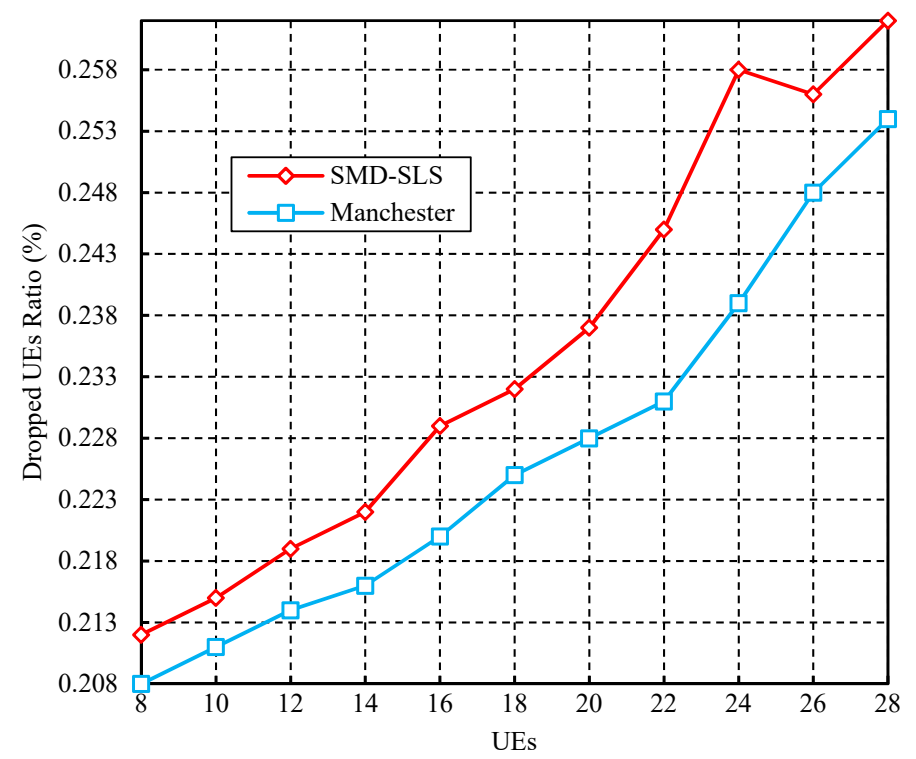

Figure 9. Dropping ratio of proposed vs Manchester methods in homogeneous network.

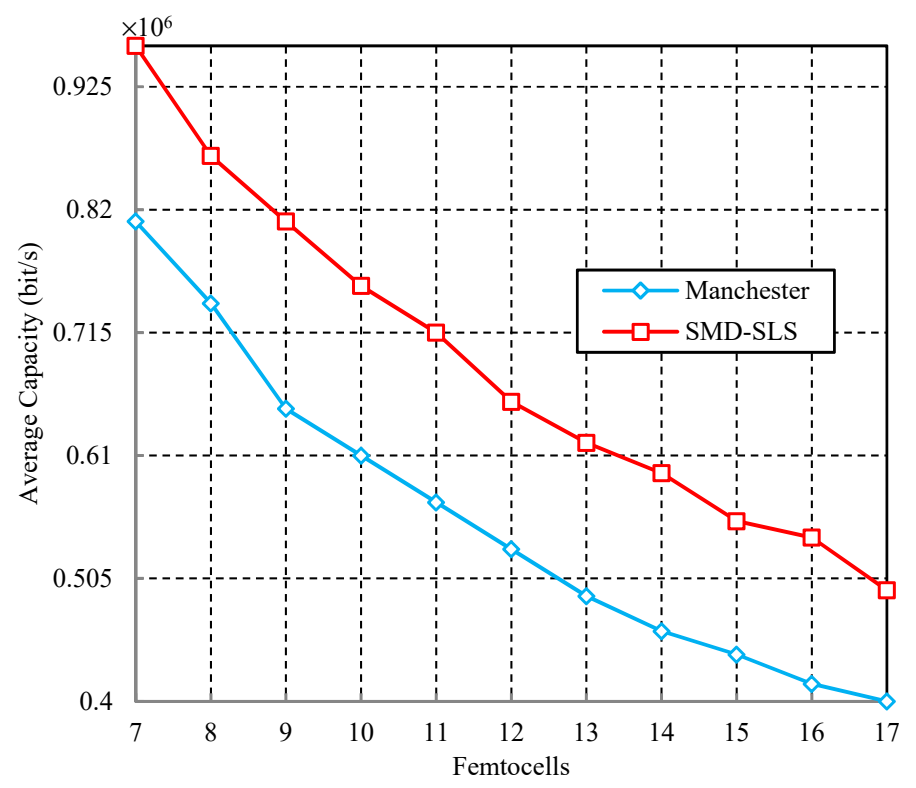

Figure 10. Average capacity for deployed femtocells in homogeneous network.

For a heterogeneous solution, the femtocell access threshold $\mu=12 \mathrm{~dB}$ offloads the macrocells with the femtocells. Figure 11 shows the average throughput vs CDF for evaluating the performance of SMD-SLS compared to Manchester and the benchmark technique. It can be inferred from the results that the performance of SMD-SLS is superior to the other techniques. The results show that the improvement in performance is less apparent in a heterogeneous network compared to a homogeneous network, since the femtocells are randomly deployed in the coverage area of the macrocells, which reduces the overlapped area by eliminating the deactivated cells. 


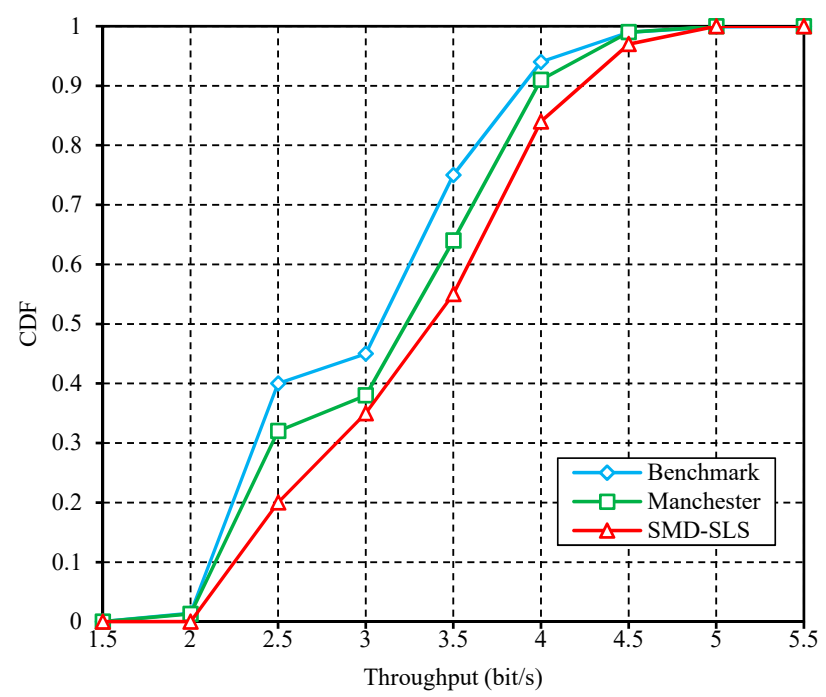

Figure 11. Average throughput vs CDF in a heterogeneous medium.

\section{Limitations, Practical Implications and Future Work}

The major limitation is the failure in deploying advanced machine learning models for the selection of sleep modes. The semi-Markov model designed cannot be applied for other network architectures and is purely limited to this model.

The throughput results for the homogeneous model and heterogonous model shows the application of the proposed method on advanced networks with high bandwidth constraints. It further can provide better adoption on small cell networks and offers wide benefits in the allocation of resources using a semi-Markov process.

In future, the proposed small cell deactivation method may be modelled with a machine learning or a heuristic model that can enhance the strategy of selecting the small cell in an optimal manner. This would improve the network throughput of the entire system.

\section{Conclusions}

A hybrid semi-Markov sleep mode selection with a small cell deactivation strategy is shown to improve the efficiency of power and resource utilization in small cell OFDMA networks. The semi-Markov process optimizes sleep mode selection, thereby minimizing the overall power consumption in networks using operational mode transitions. Network performance is increased by deactivating the small cells located at undesirable spots. Comparison with conventional non-sleeping mode cells demonstrated that the proposed hybrid model increased the efficiency of data rate and power efficiency by $38.5 \%$ and $19.2 \%$, respectively. The CDF of the proposed system demonstrated that the throughput performance is increased up to $34 \%$ compared to non-sleeping mode heterogeneous cell systems. Finally, the proposed SMD-SLS system approaches the benchmark performance with lowered computational cost.

Author Contributions: Conceptualization, N.G.V.K. and Y.N.; Data curation, K.S.; Formal analysis, Y.N.; Methodology, N.G.V.K. and R.P.; Project administration, Y.N.; Resources, N.G.V.K.; Software, K.S.; Supervision, R.P. and A.H.G.; Validation, K.S. and A.H.G.; Writing—original draft, N.G.V.K.; Writing—review \& editing, R.P. and A.H.G. All authors have read and agreed to the published version of the manuscript.

Funding: This research received no external funding.

Conflicts of Interest: The authors declare no conflict of interest. 


\section{References}

1. Pattanayak, P.; Kumar, P. Combined user and antenna scheduling scheme for MIMO-OFDM networks. Telecommun. Syst. 2019, 70, 3-12. [CrossRef]

2. Chu, X.; Lopez-Perez, D.; Yang, Y.; Gunnarsson, F. Heterogeneous Cellular Networks: Theory, Simulation and Deployment, 2nd ed.; Cambridge Press: Cambridge, UK, 2008.

3. Lopez-Perez, D.; Valcarce, A.; de la Roche, G.; Zhang, J. OFDMA femtocells: A Roadmap on Interference Avoidance. IEEE Commun. Mag. 2009, 47, 41-48. [CrossRef]

4. Pateromichelakis, E.; Shariat, M.; Quddus, A.U.; Tafazolli, R. On the Analysis of Co-tier Interference in Femtocells. In Proceedings of the IEEE 22nd International Symposium on Personal, Indoor and Mobile Radio Communications, Toronto, ON, Canada, 11-14 September 2011; pp. 122-126.

5. Ndong, M.; Fujii, T. CROSS-Tier Interference Management with a Distributed Antenna System for Multi-Tier Cellular Networks. Eurasip J. Wirel. Commun. Netw. 2014, 73, 1-12. [CrossRef]

6. Adeel, A.; Larijani, H.; Ahmadinia, A. Resource Management and Inter-Cell-Interference Coordination in LTE Uplink System Using Random Neural Network and Optimization. IEEE Access 2015, 3, 1963-1979. [CrossRef]

7. Novlan, T.D.; Ganti, R.K.; Ghosh, A.; Andrews, J.G. Analytical Evaluation of Fractional Frequency Reuse for OFDMA Cellular Networks. IEEE Trans. Wirel. Commun. 2011, 10, 4294-4305. [CrossRef]

8. Cao, D.; Zhou, S.; Niu, Z. Improving the Energy Efficiency of Two-Tier Heterogeneous Cellular Networks through Partial Spectrum Reuse. IEEE Trans. Wirel. Commun. 2013, 12, 4129-4141. [CrossRef]

9. Mukherjee, S.; Guvenc, I. Effects of Range Expansion and Interference Coordination on Capacity and Fairness in Heterogeneous Networks. In Proceedings of the 2011 Conference Record of the Forty Fifth Asilomar Conference on Signals, Systems and Computers (ASILOMAR), Pacific Grove, CA, USA, 6-9 November 2011; pp. 1855-1859.

10. Kamitsos, I.; Tsiaflakis, P.; Kerpez, K.J.; Ha, S.; Chiang, M. Stable Sleep Mode Optimization for Energy Efficient DSL. IEEE Trans. Commun. 2015, 63, 5116-5127. [CrossRef]

11. Lee, Y.L.; Chuah, T.C.; Loo, J.; Vinel, A. Recent Advances in Radio Resource Management for Heterogeneous LTE / LTE-A Networks. IEEE Commun. Surv. Tutor. 2014, 16, 2142-2180. [CrossRef]

12. Hasib, A.; Fapojuwo, A. Analysis of Common Radio Resource Management Scheme for End-to-End QoS Support in Multiservice Heterogeneous Wireless Networks. IEEE Trans. Veh. Technol. 2008, 57, 2426-2439. [CrossRef]

13. Ashraf, I.; Boccardi, F.; Ho, L. SLEEP mode techniques for small cell deployments. IEEE Commun. Mag. 2011, 49, 72-79. [CrossRef]

14. Wang, M.; Zhu, X.; Zeng, Z.; Wan, S.; Li, W. System performance analysis of OFDMA based femtocell networks. In Proceedings of the IET International Conference on Communication Technology and Application (ICCTA 2011), Beijing, China, 14-16 October 2011; pp. 1-6.

15. Kong, L.; Wong, K.W.G.; Tsang, D. Performance study and system optimization on sleep mode operation in IEEE 802.16e. IEEE Trans. Wirel. Commun. 2009, 8, 4518-4528. [CrossRef]

16. Kong, L.; Wong, K.; Tsang, D.H.W. Sleep Mode Selection Employing a Semi-Markov Decision Process. U.S. Patent US8565134B2, 8 September 2013.

17. Wang, R.; Haas, H.; Thompson, J.; Grant, P. Sleep mode design for green base stations. IET Commun. 2011, 5, 2606-2616. [CrossRef]

18. Ren, P.; Tao, M. A Decentralized Sleep Mechanism in Heterogeneous Cellular Networks With QoS Constraints. IEEE Wirel. Commun. Lett. 2014, 3, 509-512. [CrossRef]

19. Lucas-Estañ, M.C.; Gozalvez, J. On the Real-Time Hardware Implementation Feasibility of Joint Radio Resource Management Policies for Heterogeneous Wireless Networks. IEEE Trans. Mob. Comput. 2011, 12, 193-205. [CrossRef]

20. Singh, S.; Andrews, J.G. Joint Resource Partitioning and Offloading in Heterogeneous Cellular Networks. IEEE Trans. Wirel. Commun. 2013, 13, 888-901. [CrossRef]

21. Carvalho, G.H.S.; Woungang, I.; Anpalagan, A.; Hossain, E. QoS-Aware Energy-Efficient Joint Radio Resource Management in Multi-RAT Heterogeneous Networks. J. Latex Cl. Files 2012, 11, 1-12. [CrossRef] 
22. Gerasimenko, M.; Moltchanov, D.; Florea, R.; Andreev, S.; Koucheryavy, Y.; Himayat, N.; Yeh, S.; Talwar, S. Cooperative Radio Resource Management in Heterogeneous Cloud Radio Access Networks. IEEE Access 2015, 3, 397-406. [CrossRef]

23. Chu, E.; Song, N.-O.; Sung, D.K. Remaining Energy-Aware QoE Based Binary-Exponential Sleep Mode Operation. IEEE Commun. Lett. 2015, 19, 2226-2229. [CrossRef]

24. Jia, C.; Lim, T.J. Resource Partitioning and User Association with Sleep-Mode Base Stations in Heterogeneous Cellular Networks. IEEE Trans. Wirel. Commun. 2015, 14, 1. [CrossRef]

25. Chou, S.F.; Chao, H.-L.; Liu, C.-L. An Efficient Measurement Report Mechanism for Long Term Evolution Networks. In Proceedings of the IEEE Personal Indoor and Mobile Radio Communications, Toronto, ON, Canada, 11-14 September 2011; pp. 197-201.

26. Kazmi, S.M.A.; Tran, N.H.; Saad, W.; Le, L.B.; Ho, T.M.; Hong, C. Optimized Resource Management in Heterogeneous Wireless Networks. IEEE Commun. Lett. 2016, 20, 1397-1400. [CrossRef]

(C) 2020 by the authors. Licensee MDPI, Basel, Switzerland. This article is an open access article distributed under the terms and conditions of the Creative Commons Attribution (CC BY) license (http://creativecommons.org/licenses/by/4.0/). 